\title{
A LOWER BOUND ON THE GLOBAL POWERFUL ALLIANCE NUMBER IN TREES
}

\author{
Saliha OuAtiki ${ }^{1, *}$ And Mohamed Bouzefrane ${ }^{2}$
}

\begin{abstract}
For a graph $G=(V, E)$, a set $D \subseteq V$ is a dominating set if every vertex in $V-D$ is either in $D$ or has a neighbor in $D$. A dominating set $D$ is a global offensive alliance (resp. a global defensive alliance) if for each vertex $v$ in $V-D$ (resp. $v$ in $D$ ) at least half the vertices from the closed neighborhood of $v$ are in $D$. A global powerful alliance is both global defensive and global offensive. The global powerful alliance number $\gamma_{p a}(G)$ is the minimum cardinality of a global powerful alliance of $G$. We show that if $T$ is a tree of order $n$ with $l$ leaves and $s$ support vertices, then $\gamma_{p a}(T) \geq \frac{3 n-2 l-s+2}{5}$. Moreover, we provide a constructive characterization of all extremal trees attaining this bound.
\end{abstract}

Mathematics Subject Classification. 05C69, $05 \mathrm{C} 05$.

Received May 18, 2020. Accepted February 20, 2021.

\section{INTRODUCTION}

Let $G=(V, E)$ be a finite and simple graph of order $n$. The open neighborhood of a vertex $v$ is $N(v)=$ $\{u \in V \mid u v \in E\}$ and the closed neighborhood of $v$ is $N[v]=N(v) \cup\{v\}$. The degree of $v$, denoted by $d_{G}(v)$, is the size of its open neighborhood. For a vertex $v$, the eccentricity of $v$ is the maximum of the distance to any vertex in the graph. The diameter of $G$ noted $\operatorname{diam}(G)$ is the maximum of the eccentricity of any vertex in the graph. A vertex of degree one is called a pendant vertex or a leaf and its neighbor is called a support vertex. A support vertex with exactly one non-leaf neighbor is called a pendant support vertex. If $v$ is a support vertex, then $L_{v}$ will denote the set of the leaves attached at $v$. A support vertex $v$ is said to be strong if $\left|L_{v}\right| \geq 2$ and weak otherwise. We denote the set of leaves of a graph $G$ by $L(G)$ and the set of support vertices by $S(G)$, and let $|L(G)|=l(G),|S(G)|=s(G)$ (we use $l, s$ if there is no ambiguity). We write $P_{n}$ for the path of order $n$. If a tree $T=P_{2}$, we consider without loss of generality that $P_{2}$ has one support vertex and one leaf, so $l\left(P_{2}\right)=s\left(P_{2}\right)=1$. A star $S_{p}$ is the complete bipartite graph $K_{1, p}$. A tree containing exactly two non-pendant vertices is called a double star. A double star with respectively $p$ and $q$ leaves attached at each support vertex is denoted by $S_{p, q}$. Denote by $T_{x}$ the subtree induced by a vertex $x$ and its descendants in a rooted tree $T$.

In [8], Hedetniemi et al. introduced several types of alliances in graphs including the offensive and the defensive alliances and the powerful alliances we consider here. A dominating set $D$ of $G$ is called a global offensive alliance (resp. a global defensive alliance) if for each vertex $v$ in $V-D$ (resp. $v$ in $D$ ) at least half the vertices from

Keywords. Domination, global powerful alliance, trees.

1 Department of Mathematics, University of Boumerdes, Boumerdes, Algeria.

2 Department of Mathematics, B.P. 270, University of Blida, Blida, Algeria.

*Corresponding author: saliha_ouatiki@yahoo.fr, ouatik.s@univ-boumerdes.dz 
the closed neighborhood of $v$ are in $D:|N[v] \cap D| \geq|N[v]-D|$. Alliances in graph were studied in [1-9]. A global powerful alliance is both global defensive and global offensive which is equivalent to saying that for every vertex $v$ in $V,|N[v] \cap D| \geq|N[v]-D|$. The global powerful alliance number $\gamma_{p a}(G)$ is the minimum cardinality of a global powerful alliance. The entire vertex set is both a global offensive alliance and a global defensive alliance for any graph $G$, so every graph $G$ has a global powerful alliance number. Note that this parameter has been little studied in literature since its introduction. We abbreviate global powerful alliance as gpa. A gpa with minimum cardinality $\gamma_{p a}(G)$ is called a $\gamma_{p a}(G)$-set. A lower bound for a global powerful alliance number for any tree with equal powerful alliance and global powerful alliance numbers was given in [2] in term of the maximum degree $\triangle(G)$. They show that $\gamma_{p a}(T) \geq\left\lceil\frac{\Delta(T)+1}{2}\right\rceil$ and they characterize all such trees achieving this bound. In [3], Cami et al. have proved that finding an optimal global powerful (offensive, defensive) alliance is an NP-complete problem. However, a linear time algorithm that finds the smallest global powerful alliance of any weighted tree was given in [7].

\section{LOWER BOUND}

We begin by giving the global powerful alliance number of a tree $T$, where $T$ is either a star or a double star.

Observation 2.1. If $T$ is a star $S_{p}$, then $\gamma_{p a}\left(S_{p}\right)=\left\lceil\frac{p+1}{2}\right\rceil$.

Observation 2.2. If $T$ is a double star $S_{p, q}$, then $\gamma_{p a}\left(S_{p, q}\right)=\left\lfloor\frac{p+1}{2}\right\rfloor+\left\lfloor\frac{q+1}{2}\right\rfloor$.

The following observation about a support in a $\gamma_{p a}(G)$-set will be useful.

Observation 2.3. If $G$ is a connected graph of order at least three, then there exists a $\gamma_{p a}(G)$-set that contains all support vertices.

Proof. If a $\gamma_{p a}(G)$-set, $D$ does not contain a support vertex $u$, then $D$ contains all leaves of $u$. So, we can replace any leaf of $u$ by $u$ in $D$.

We now present our main result of this section.

Theorem 2.4. Let $T$ be a tree of order $n$ with $l$ leaves and $s$ support vertices. Then $\gamma_{p a}(T) \geq \frac{3 n-2 l-s+2}{5}$.

Proof. We proceed by induction on the order of $T$. Clearly, the result holds for $1 \leq n \leq 3$ where $T$ is $P_{n}$ and thus $\gamma_{p a}(T) \geq \frac{3 n-2 l-s+2}{5}$. Let $n \geq 4$ and assume that every tree $T^{\prime}$ of order $n^{\prime}, 4 \leq n^{\prime}<n$ with $l^{\prime}$ leaves and $s^{\prime}$ support vertices verifies $\gamma_{p a}\left(T^{\prime}\right) \geq \frac{3 n^{\prime}-2 l^{\prime}-s^{\prime}+2}{5}$. Let $T$ be a tree with $l$ leaves and $s$ support vertices. If $\operatorname{diam}(T)=2$, then $T$ is a star $S_{p}$ and from Observation 2.1, $\gamma_{p a}\left(S_{p}\right)=\left\lceil\frac{p+1}{2}\right\rceil$. Since, $n=p+1, s=1$ and $l=p$, we get $\gamma_{p a}(T)>\frac{3 n-2 l-s+2}{5}=\frac{p+4}{5}$. If $\operatorname{diam}(T)=3$, then $T=S_{p, q}$ and from Observation 2.2, $\gamma_{p a}\left(S_{p, q}\right)=\left\lfloor\frac{p+1}{2}\right\rfloor+\left\lfloor\frac{q+1}{2}\right\rfloor$. Since $n=p+q+2, l=p+q$ and $s=2$, we obtain $\gamma_{p a}\left(S_{p, q}\right) \geq \frac{p+q+6}{5}=\frac{3 n-2 l-s+2}{5}$ and hence the result is valid. Assume that $\operatorname{diam}(T)=t \geq 4$. Let $T$ be rooted at a leaf $u_{t}$ of a maximum eccentricity, that is $\operatorname{ecc}\left(u_{t}\right)=\operatorname{diam}(T)$ and let $u_{1}$ be a support vertex at distance $\operatorname{diam}(T)-1$ from $u_{t}$. Let $u_{i+1}, 0 \leq i \leq t-1$ be the parent of $u_{i}$ in the rooted tree. Let $P: u_{0}, u_{1}, \ldots, u_{t}$ be then the resultant diametral path. It's clear that $u_{1}$ is a pendant support vertex. Let $D$ be a $\gamma_{p a}(T)$-set with a fewest possible number of leaves. Consider the following cases.

Case 1. $\left|L_{u_{1}}\right|=k \geq 3$. By the choice of $D$, both vertices $u_{1}$ and $u_{2}$ are in $D$ and $D$ contains $\left\lfloor\frac{k-1}{2}\right\rfloor$ leaves of $u_{1}$. Let $u^{\prime}$ be any leaf of $u_{1}$ not in $D$. Let $T^{\prime}=T-\left(L_{u_{1}}-u^{\prime}\right)$ (see Fig. 1), then $n^{\prime}=n-k+1, l^{\prime}=l-k+1$ and $s^{\prime}=s$. Since, $\operatorname{diam}(T) \geq 4$ then $n^{\prime} \geq 5$. Clearly $D \cap V\left(T^{\prime}\right)$ is a gpa of $T^{\prime}$ and so $\gamma_{p a}\left(T^{\prime}\right) \leq \gamma_{p a}(T)-\left\lfloor\frac{k-1}{2}\right\rfloor$. Using the inductive hypothesis on $T^{\prime}$, we get $\gamma_{p a}(T) \geq \frac{3 n^{\prime}-2 l^{\prime}-s^{\prime}+2}{5}+\left\lfloor\frac{k-1}{2}\right\rfloor$. Since $n^{\prime}=n-k+1, l^{\prime}=l-k+1$ and $s^{\prime}=s$ then $\gamma_{p a}(T) \geq \frac{3 n-2 l-s+2}{5}+\frac{1-k}{5}+\left\lfloor\frac{k-1}{2}\right\rfloor>\frac{3 n-2 l-s+2}{5}$. 


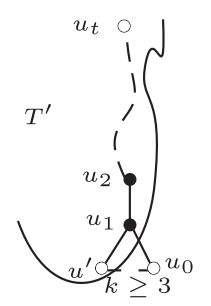

FiguRE 1. $T^{\prime}=T-\left(L_{u_{1}}-u^{\prime}\right)$.

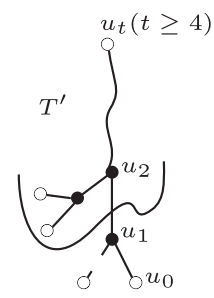

(1)

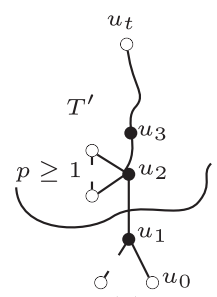

(2)

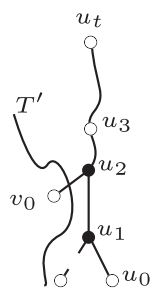

(1)

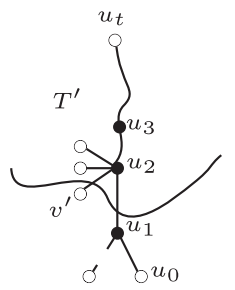

(2)

(a) $:\left|N\left[u_{2}\right] \cap D\right|>\left|N\left[u_{2}\right]-D\right|$.

(b) : $\left|N\left[u_{2}\right] \cap D\right|=\left|N\left[u_{2}\right]-D\right|$.

Figure 2. The black vertices are in $D$. In (a), $T^{\prime}=T-T_{u_{1}}$. In (b), $T^{\prime}=T-\left\{v_{0}\right\}$ or $T^{\prime}=T-\left(T_{u_{1}} \cup\left\{v^{\prime}\right\}\right)$.

Case 2. $\left|L_{u_{1}}\right|=k \leq 2$ and $D \cap L\left(u_{1}\right)=\emptyset$. Any child of $u_{2}$ is either a leaf or a support vertex. By Observation 2.3 and the minimality of $D$, both $u_{1}$ and $u_{2}$ are in $D$.

Subcase 2.1 $d_{T}\left(u_{2}\right) \geq 3$. Assume $u_{2}$ is a support vertex with $\left|L_{u_{2}}\right|=p \geq 2$ or $u_{2}$ has a child which is a support vertex. Then, if $\left|N\left[u_{2}\right] \cap D\right|>\left|N\left[u_{2}\right]-D\right|$, let us consider $T^{\prime}=T-T_{u_{1}}$ (see (a) in Fig. 2). As $\operatorname{diam}(T) \geq 4$, then $n^{\prime} \geq 4$. It is obvious that $D \cap V\left(T^{\prime}\right)$ is a gpa of $T^{\prime}$ implying that $\gamma_{p a}\left(T^{\prime}\right) \leq \gamma_{p a}(T)-1$. Hence, $\gamma_{p a}(T) \geq \gamma_{p a}\left(T^{\prime}\right)+1$. Using the inductive hypothesis on $T^{\prime}$, we obtain $\gamma_{p a}(T) \geq \frac{3 n^{\prime}-2 l^{\prime}-s^{\prime}+2}{5}+1$. Since $n^{\prime}=n-1-k, l^{\prime}=l-k$ and $s^{\prime}=s-1$, we get $\gamma_{p a}(T) \geq \frac{3 n-2 l-s+2}{5}+\frac{3-k}{5}>\frac{3 n-2 l-s+2}{5}$. Suppose now that $u_{2}$ is a support vertex with $\left|N\left[u_{2}\right] \cap D\right|=\left|N\left[u_{2}\right]-D\right|$. Let $v^{\prime}$ be a leaf-neighbor of $u_{2}$ not in $D$ and let $T^{\prime}=T-\left(T_{u_{1}} \cup\left\{v^{\prime}\right\}\right)$ (see (b.2) in Fig. 2). Clearly, $D \cap V\left(T^{\prime}\right)$ is a gpa of $T^{\prime}$ which implies that $\gamma_{p a}\left(T^{\prime}\right) \leq \gamma_{p a}(T)-1$. Using the inductive hypothesis on $T^{\prime}$, we get $\gamma_{p a}(T) \geq \frac{3 n^{\prime}-2 l^{\prime}-s^{\prime}+2}{5}+1$. Since $n^{\prime}=n-2-k, l^{\prime}=l-k-1$ and $s^{\prime}=s-1$, we obtain $\gamma_{p a}(T) \geq \frac{3 n-2 l-s+2}{5}+\frac{2-k}{5} \geq \frac{3 n-2 l-s+2}{5}$.

Suppose now that $u_{2}$ is a support vertex with $\left|L_{u_{2}}\right|=1$ and $d_{T}\left(u_{2}\right)=3$ (see (a.2) in Fig. 2). If $u_{3} \in D$, then we consider $T^{\prime}=T-T_{u_{1}}$. As before, it can be seen that $\gamma_{p a}(T)>\frac{3 n-2 l-s+2}{5}$. Thus, assume that $u_{3} \notin D$. Hence, $u_{3}$ is not a support vertex according to the Observation 2.3. Let $v_{0}$ be the unique leaf of $u_{2}$ in $T$ and let us consider $T^{\prime}=T-\left\{v_{0}\right\}$ (see (b.1) in Fig. 2). We have $\operatorname{diam}\left(T^{\prime}\right) \geq 4$ and so $n^{\prime} \geq 5$. The set $D \cap V\left(T^{\prime}\right)$ is a gpa of $T^{\prime}$ implying that $\gamma_{p a}\left(T^{\prime}\right) \leq \gamma_{p a}(T)$. Using the inductive hypothesis on $T^{\prime}$ and since $n^{\prime}=n-1, l^{\prime}=l-1$ and $s^{\prime}=s-1$, we obtain $\gamma_{p a}(T) \geq \frac{3 n-2 l-s+2}{5}$.

Subcase $2.2 d_{T}\left(u_{2}\right)=2$. Suppose $u_{3} \in D$. Any child of $u_{3}$ is either a leaf or a support vertex or a non-leaf neighbor of a support vertex. According to Observation 2.3, and the minimality of $D$, any non-leaf neighbor of $u_{3}$ is in $D$. Assume that $\left|N\left[u_{3}\right] \cap D\right|>\left|N\left[u_{3}\right]-D\right|$ (see (a.1) in Fig. 3). Let us consider $T^{\prime}=T-T_{u_{2}}$. It is easy to check that if $T^{\prime}=K_{2}$ then $n=k+4, l=k+1$ and $s=2$ and so $\gamma_{p a}(T)=3>\frac{3 n-2 l-s+2}{5}=\frac{k+10}{5}$ and the result holds. Further, $T^{\prime} \neq S_{2}$ otherwise $\left|N\left[u_{3}\right] \cap D\right|=\left|N\left[u_{3}\right] \cap(V-D)\right|$. Thus $n^{\prime} \geq 4$ and $D \cap V\left(T^{\prime}\right)$ is a gpa of $T^{\prime}$ implying that $\gamma_{p a}\left(T^{\prime}\right) \leq \gamma_{p a}(T)-2$. Using the inductive hypothesis on $T^{\prime}$ and since $n^{\prime}=n-2-k, l^{\prime} \leq l-k+1$ 


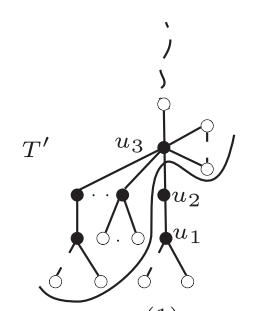

(1)

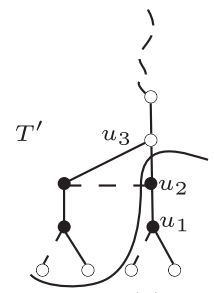

(2)

(a) : $\left|N\left[u_{3}\right] \cap D\right|>\left|N\left[u_{3}\right]-D\right|$.

FiguRE 3. In (a): either $u_{3}$ is in $D$ or not, we consider $T^{\prime}=T-T_{u_{2}}$.

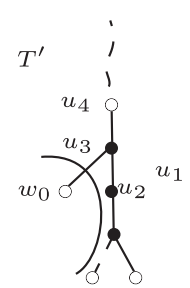

(1)

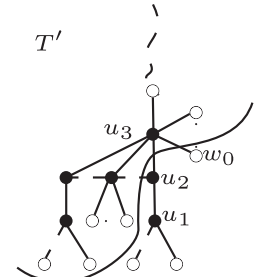

(2)

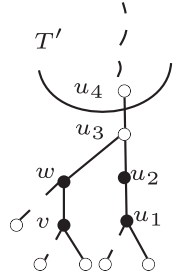

(3)

(b) $:\left|N\left[u_{3}\right] \cap D\right|=\left|N\left[u_{3}\right]-D\right|$.

Figure 4. In (b), if $u_{3} \in D, T^{\prime}=T-\left\{w_{0}\right\}$ if $d_{T}\left(u_{3}\right)=3$, otherwise, $T^{\prime}=T-\left(T_{u_{2}} \cup\left\{w_{0}\right\}\right)$. If $u_{3} \notin D, T^{\prime}=T-T_{u_{3}}$.

and $s^{\prime} \leq s$, we obtain $\gamma_{p a}(T) \geq \frac{3 n-2 l-s+2}{5}+\frac{2-k}{5} \geq \frac{3 n-2 l-s+2}{5}$. Let us remark that the case when $d_{T}\left(u_{3}\right)=2$ is included in this case.

Suppose now that $\left|N\left[u_{3}\right] \cap D\right|=\left|N\left[u_{3}\right]-D\right|$. If $d_{T}\left(u_{3}\right)=3$, then $\left|L_{u_{3}}\right|=1$ and $u_{4}$ is not in $D$ (see (b.1) in Fig. 4). Let $w_{0}$ be the unique leaf of $u_{3}$ and let us consider $T^{\prime}=T-\left\{w_{0}\right\}$. As $\operatorname{diam}(T) \geq 4$ then $n^{\prime} \geq 5$. Clearly, $D \cap V\left(T^{\prime}\right)$ is a gpa of $T^{\prime}$ implying that $\gamma_{p a}\left(T^{\prime}\right) \leq \gamma_{p a}(T)$. Using the inductive hypothesis on $T^{\prime}$ and since $n^{\prime}=n-1, l^{\prime}=l-1$ and $s^{\prime}=s-1$, we obtain $\gamma_{p a}(T) \geq \frac{3 n-2 l-s+2}{5}$.

Suppose now that $d_{T}\left(u_{3}\right)>3$. So, $\left|L_{u_{3}}\right| \geq 2$ and $u_{3}$ has necessarily a leaf say $w_{0} \notin D$. Let us consider $T^{\prime}=T-\left(T_{u_{2}} \cup\left\{w_{0}\right\}\right)$ (see (b.2) in Fig. 4). If $u_{4} \in D$ then $\left|L_{u_{3}}\right| \geq 3$ and so $n^{\prime} \geq 4$. Otherwise, $u_{4} \notin D,\left|L_{u_{3}}\right| \geq 2$ and $L_{u_{3}} \cap D=\emptyset$. Thus, $u_{3}$ has a non-leaf child in $D \cap V\left(T^{\prime}\right)$ and then $n^{\prime} \geq 4$. Clearly, $D \cap V\left(T^{\prime}\right)$ is a gpa of $T^{\prime}$ implying that $\gamma_{p a}\left(T^{\prime}\right) \leq \gamma_{p a}(T)-2$. Using the inductive hypothesis on $T^{\prime}$ and since $n^{\prime}=n-3-k, l^{\prime}=l-1-k$ and $s^{\prime}=s-1$, we obtain $\gamma_{p a}(T) \geq \frac{3 n^{\prime}-2 l^{\prime}-s^{\prime}+2}{5}+2 \geq \frac{3 n-2 l-s+2}{5}+\frac{4-k}{5}$. Since $k \leq 2$ then we obtain $\gamma_{p a}(T)>$ $\frac{3 n-2 l-s+2}{5}$. Suppose now that $u_{3} \notin D$. Then $u_{3}$ is different from a support vertex according to Observation 2.3. Further, $u_{3}$ cannot be the unique non-leaf neighbor of a support vertex as it can replace any leaf in $D$ of its support neighbor which is a contradiction. So, any neighbor of $u_{3}$ is in $D$. If $\left|N\left[u_{3}\right] \cap D\right|>\left|N\left[u_{3}\right]-D\right|$ (see (a.2) in Fig. 3). Let us consider $T^{\prime}=T-T_{u_{2}}$ and we proceed with the same manner as previously (the case $d_{T}\left(u_{3}\right)=2$ is included here). Assume now that $\left|N\left[u_{3}\right] \cap D\right|=\left|N\left[u_{3}\right]-D\right|$. So, $d_{T}\left(u_{3}\right)=3$ and $u_{4}$ is not in $D$ (see (b.3) in Fig. 4). Let $w, v$ be the children of $u_{3}$ and $w$, respectively in $T_{u_{3}}$ and let us consider $T^{\prime}=T-T_{u_{3}}$. If $w$ is a support vertex then $\left|L_{w}\right|=1$ otherwise, $u_{3}$ may replace a leaf of $w$ in $D$ which is a contradiction. Set $\left|L_{v}\right|=p$. By analogy to $L_{u_{1}}, p \leq 2$. As $u_{4}, u_{3} \notin D$, then $u_{4}$ has at least two neighbors in $D \cap V\left(T^{\prime}\right)$. The vertex $u_{t}$ cannot be a parent of $u_{4}$ otherwise $u_{4}$ is a support vertex and then $u_{4}$ will be in $D$ which is a contradiction. So, $n^{\prime} \geq 4$. It is obvious that $D \cap V\left(T^{\prime}\right)$ is a gpa of $T^{\prime}$ implying that $\gamma_{p a}\left(T^{\prime}\right) \leq \gamma_{p a}(T)-4$. Using inductive hypothesis on $T^{\prime}$ and since $n^{\prime} \geq n-6-p-k, l^{\prime} \leq l-p-k$ and $s^{\prime} \leq s-2$, we obtain $\gamma_{p a}(T) \geq \frac{3 n-2 l-s+2}{5}+\frac{4-(p+k)}{5}$. As $p+k \leq 4$ then we get $\gamma_{p a}(T) \geq \frac{3 n-2 l-s+2}{5}$ and the result holds. 


\section{TREES WITH $\gamma_{p a}(T)=\frac{3 n-2 l-s+2}{5}$}

In this section, we characterize the extremal trees attaining the bound given in the Theorem 2.4. For the purpose, we define a family $\mathfrak{F}$ of all trees $T$ that can be obtained from the sequence $T_{1}, T_{2}, \ldots, T_{k}(k \geq 1)$ of trees, where $T_{1}$ is the path $P_{2}, T=T_{k}$, and if $k \geq 2, T_{i+1}$ is obtained recursively from $T_{i}$ by the operation listed below.

- Operation $O$ : Assume $u$ is a support vertex of $T_{i}$. Then the tree $T_{i+1}$ is obtained from $T_{i}$ by adding one leaf by attachment edge to $u$ and by adding a path $P_{3}: x y z$ by joining $u$ to $y$.

\subsection{Preliminary results}

From the way in which a tree $T \in \mathfrak{F}$ is constructed, we make the following observation.

Observation 3.1. Let $T$ be a tree of $\mathfrak{F}$ different from $P_{2}$. Then every non-leaf vertex of $T$ is a strong support vertex.

Theorem 3.2. Let $T$ be a nontrivial tree with l leaves and s support vertices. If $T \in \mathfrak{F}$ then $\gamma_{p a}(T)=\frac{3 n-2 l-s+2}{5}$.

Proof. Let $T$ be a tree of $\mathfrak{F}$. We use induction on the number of operations $O$ performed to construct $T$. Clearly, if $T=T_{1}=P_{2}$ then $\gamma_{p a}(T)=1=\frac{3 n-2 l-s+2}{5}$. Assume that the property is true for all trees of $\mathfrak{F}$ constructed with $k-1 \geq 0$ operations, and let $T$ be a tree of $\mathfrak{F}$ constructed with $k$ operations. Thus $T$ is obtained by performing the operation $O$ on a tree $T^{\prime}=T_{k-1} \in \mathfrak{F}$ of order $n^{\prime}$ with $l^{\prime}$ leaves and $s^{\prime}$ support vertices. By the induction hypothesis, we have $\gamma_{p a}\left(T^{\prime}\right)=\frac{3 n^{\prime}-2 l^{\prime}-s^{\prime}+2}{5}$. By Observation 2.3, there exist a $\gamma_{p a}\left(T^{\prime}\right)$-set that contains the support vertex $u$. Such a set can be extend to a gpa of $T$ by adding $y$. Then, $\gamma_{p a}(T) \leq \gamma_{p a}\left(T^{\prime}\right)+1=\frac{3 n^{\prime}-2 l^{\prime}-s^{\prime}+2}{5}+1$. On the other hand, let $D$ be a $\gamma_{p a}(T)$-set with a fewest possible number of leaves. So, by the minimality of $D$ and Observation 2.3, both $u$ and $y$ are in $D$. Let $u^{\prime}$ be the leaf of $u$ in $T-T^{\prime}$. If $u^{\prime} \notin D$ then $D \cap V\left(T^{\prime}\right)$ is a gpa of $T^{\prime}$. Otherwise, we replace $u^{\prime} \in D$ by any leaf of $u$ in $T^{\prime}$ not in $D$. It follows then that $D \cap V\left(T^{\prime}\right)$ is a gpa of $T^{\prime}$ implying that $\gamma_{p a}\left(T^{\prime}\right) \leq \gamma_{p a}(T)-1$. We deduce then that $\gamma_{p a}(T)=\gamma_{p a}\left(T^{\prime}\right)+1=\frac{3 n^{\prime}-2 l^{\prime}-s^{\prime}+2}{5}+1$. Since $n^{\prime}=n-4, l^{\prime}=l-3$ and $s^{\prime}=s-1$, we get $\gamma_{p a}(T)=\frac{3 n-2 l-s+2}{5}$.

\subsection{The main result}

Theorem 3.3. Let $T$ be a nontrivial tree with $l$ leaves and s support vertices. Then $\gamma_{p a}(T)=\frac{3 n-2 l-s+2}{5}$ if and only if $T \in \mathfrak{F}$.

Proof. The sufficiency follows from Theorem 3.2. To prove the necessity, we proceed by induction on the order $n$ of a tree $T$ verifying $\gamma_{p a}(T)=\frac{3 n-2 l-s+2}{5}$. We shall prove that $T \in \mathfrak{F}$. If $\operatorname{diam}(T)=1$, then $T=K_{2} \in \mathfrak{F}$. If $\operatorname{diam}(T)=2$ then $T$ is a star $S_{p}$ with $p \geq 2$ and from Observation $2.1, \gamma_{p a}(T)=\left\lceil\frac{p+1}{2}\right\rceil>\frac{p+4}{5}=\frac{3 n-2 l-s+2}{5}$. If $\operatorname{diam}(T)=3$ then $T$ is a double star $S_{p, q}$ and by Observation 2.2, $\gamma_{p a}(T)=\left\lfloor\frac{p+1}{2}\right\rfloor+\left\lfloor\frac{q+1}{2}\right\rfloor=\frac{p+q+6}{5}=\frac{3 n-2 l-s+2}{5}$ if and only if $p=q=2$ and $S_{2,2} \in \mathfrak{F}$. So, $\operatorname{diam}(T) \geq 4$. Suppose now that every tree $T^{\prime}$ of order $n^{\prime}, 5 \leq n^{\prime}<n$ with $l^{\prime}$ leaves and $s^{\prime}$ support vertices such that $\gamma_{p a}\left(T^{\prime}\right)=\frac{3 n^{\prime}-2 l^{\prime}-s^{\prime}+2}{5}$ is in $\mathfrak{F}$.

If any non-pendant support vertex, say $t$ of $T$ is weak, then let $T^{\prime}$ be the tree obtained from $T$ by removing a leaf, say $t^{\prime}$ adjacent to $t$. So, any $\gamma_{p a}(T)$-set with a fewest possible number of leaves is a gpa of $T^{\prime}$ implying that $\gamma_{p a}\left(T^{\prime}\right) \leq \frac{3 n-2 l-s+2}{3}$. Since $n=n^{\prime}+1, l=l^{\prime}+1$ and $s=s^{\prime}+1$ we get $\gamma_{p a}\left(T^{\prime}\right) \leq \frac{3 n^{\prime}-2 l^{\prime}-s^{\prime}+2}{3}$. The equality holds by Theorem 2.4 and consequently by using the inductive hypothesis, we deduce that $T^{\prime} \in \mathfrak{F}$. By Observation 3.1, we deduce that $t$ is a strong support vertex of $T^{\prime}$ as it is different from a leaf which is a contradiction. Henceforth, we can assume that any non-pendant support vertex of $T$ is strong.

Let $\operatorname{diam}(T)=t$ and let $P: u_{0}, u_{1}, \ldots, u_{t},(t \geq 4)$ be a diametral path and $\operatorname{root} T$ at $u_{t}$. Clearly $u_{1}$ is a pendant support vertex. Let $D$ be any $\gamma_{p a}(T)$-set that contains the fewest possible number of leaves. 


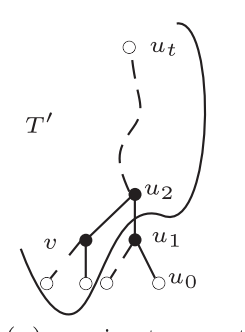

(a) : $u_{2}$ is not a support.

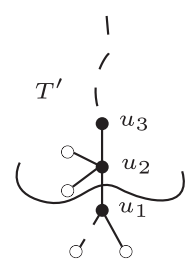

(b) $:\left|L_{u_{2}}\right|=2$.

Figure 5. In both cases (a) and (b), $T^{\prime}=T-T_{u_{1}}$.

Claim 3.4. $\left|L_{u_{1}}\right| \leq 2$.

Proof. Suppose $\left|L_{u_{1}}\right|=k \geq 3$. By the choice of $D$, both vertices $u_{1}$ and $u_{2}$ are in $D$ and $D$ contains $\left\lfloor\frac{k-1}{2}\right\rfloor$ leaves of $u_{1}$. Let $u^{\prime}$ be any leaf of $u_{1}$ not in $D$. Let $T^{\prime}=T-\left(L_{u_{1}}-\left\{u^{\prime}\right\}\right)$ (see Fig. 1). As $\operatorname{diam}(T) \geq 4$ and $k-1 \geq 2$ then $n^{\prime} \geq 5$. Clearly, $D \cap V\left(T^{\prime}\right)$ is a gpa of $T^{\prime}$ and so $\gamma_{p a}\left(T^{\prime}\right) \leq \gamma_{p a}(T)-\left\lfloor\frac{k-1}{2}\right\rfloor=\frac{3 n-2 l-s+2}{5}-\left\lfloor\frac{k-1}{2}\right\rfloor$. Since $n=n^{\prime}+k-1, l=l^{\prime}+k-1$ and $s=s^{\prime}$, we obtain $\gamma_{p a}\left(T^{\prime}\right) \leq \frac{3 n^{\prime}-2 l^{\prime}-s^{\prime}+2}{5}+\frac{k-1}{5}-\left\lfloor\frac{k-1}{2}\right\rfloor<\frac{3 n^{\prime}-2 l^{\prime}-s^{\prime}+2}{5}$ which contradicts Theorem 2.4 .

By Claim 3.4 and the choice of $D$, both $u_{1}$ and $u_{2}$ are in $D$ and $D \cap L\left(u_{1}\right)=\emptyset$. Consider the following cases. Case 1. $d_{T}\left(u_{2}\right) \geq 3$.

Claim 3.5. $u_{2}$ is a support vertex.

Proof. Any child of $u_{2}$ is either a leaf or a support vertex. Suppose $u_{2}$ has a neighbor say $v$ which is a support vertex (see (a) in Fig. 5). By Claim 3.4, $\left|L_{v}\right| \leq 2$ and so $\left\{u_{1}, u_{2}, v\right\} \subseteq D$. Let us consider $T^{\prime}=T-T_{u_{1}}$. So $\operatorname{diam}\left(T^{\prime}\right) \geq 4$ and then $n^{\prime} \geq 5$. Obviously, $D \cap V\left(T^{\prime}\right)$ is a gpa of $T^{\prime}$ implying that $\gamma_{p a}\left(T^{\prime}\right) \leq \gamma_{p a}(T)-1=$ $\frac{3 n-2 l-s+2}{5}-1$. Since $n=n^{\prime}+1+\left|L_{u_{1}}\right|, l=l^{\prime}+\left|L_{u_{1}}\right|$ and $s=s^{\prime}+1$, we obtain $\gamma_{p a}\left(T^{\prime}\right) \leq \frac{3 n^{\prime}-2 l^{\prime}-s^{\prime}+2}{5}+\frac{\left|L_{u_{1}}\right|-3}{5} \leq$ $\frac{3 n^{\prime}-2 l^{\prime}-s^{\prime}+2}{5}-\frac{1}{5}<\frac{3 n^{\prime}-2 l^{\prime}-s^{\prime}+2}{5}$ which contradicts Theorem 2.4.

Claim 3.6. $\left|L_{u_{2}}\right| \geq 3$.

Proof. By Claim 3.5, $u_{2}$ is a non-pendant support vertex, then by the remark given above, $u_{2}$ is a strong support vertex. Assume that $\left|L_{u_{2}}\right|=2$. By the minimality of $D$ and Observation 2.3, $\left\{u_{1}, u_{2}, u_{3}\right\} \subseteq D$. Let us consider $T^{\prime}=T-T_{u_{1}}$ (see (b) in Fig. 5). As $\operatorname{diam}(T) \geq 4$ and $\left|L_{u_{2}}\right|=2$ then $n^{\prime} \geq 5$. We proceed with the same manner as in the proof of Claim 3.5 and we get a contradiction.

Claim 3.7. $\left|L_{u_{1}}\right|=2$.

Proof. By Claim 3.4, $\left|L_{u_{1}}\right| \leq 2$. Suppose $\left|L_{u_{1}}\right|=1$. By the minimality of $D$, Observation 2.3 and Claim 3.6, $D$ contains $\left\{u_{1}, u_{2}, u_{3}\right\}$ and $\left\lfloor\frac{\left|L_{u_{2}}\right|-2}{2}\right\rfloor$ leaves of $u_{2}$. Since $u_{2} \in D$ and $D$ is a defensive alliance, then $\left|N\left[u_{2}\right] \cap D\right| \geq$ $\left|N\left[u_{2}\right]-D\right|$ (see Fig. 6). Suppose this inequality is strict and let us consider $T^{\prime}=T-T_{u_{1}}$ (see (a) in Fig. 6). We get a contradiction with the same manner as in the proof of the Claim 3.5.

Thus, $\left|N\left[u_{2}\right] \cap D\right|=\left|N\left[u_{2}\right]-D\right|$. Let $v$ be a leaf of $u_{2}$ not in $D$. Let us consider then $T^{\prime}=T-T_{u_{1}}-\{v\}$ (see (b) in Fig. 6). As $\operatorname{diam}(T) \geq 4$ and $\left|L_{u_{2}}\right| \geq 3$, then $n^{\prime} \geq 5$. Clearly, $D \cap V\left(T^{\prime}\right)$ is a gpa of $T^{\prime}$ implying that $\gamma_{p a}\left(T^{\prime}\right) \leq \gamma_{p a}(T)-1=\frac{3 n-2 l-s+2}{5}-1$. Since $n=n^{\prime}+2+\left|L_{u_{1}}\right|, l=l^{\prime}+1+\left|L_{u_{1}}\right|$ and $s=s^{\prime}+1$, we deduce that $\gamma_{p a}\left(T^{\prime}\right) \leq \frac{3 n^{\prime}-2 l^{\prime}-s^{\prime}+2}{5}+\frac{\left|L_{u_{1}}\right|-2}{5}$. Since $\left|L_{u_{1}}\right|=1$, then $\gamma_{p a}\left(T^{\prime}\right)<\frac{3 n^{\prime}-2 l^{\prime}-s^{\prime}+2}{5}$ which contradicts Theorem 2.4. 


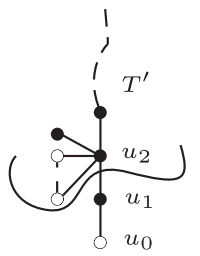

(a) : $\left|N\left[u_{2}\right] \cap D\right|>\left|N\left[u_{2}\right]-D\right|$.

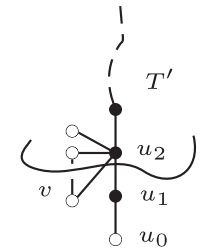

(b) : $\left|N\left[u_{2}\right] \cap D\right|=\left|N\left[u_{2}\right]-D\right|$.

Figure 6. Case $\left|L_{u_{1}}\right|=1$. In (a): $T^{\prime}=T-T_{u_{1}}$, in (b): $T^{\prime}=T-T_{u_{1}}-\{v\}$.

So, by Claim 3.7 and its proof, $\left|L_{u_{1}}\right|=2$ and $\left|N\left[u_{2}\right] \cap D\right|=\left|N\left[u_{2}\right]-D\right|$. Let then $v$ be a leaf of $u_{2}$ not in $D$ and let us consider then $T^{\prime}=T-T_{u_{1}}-\{v\}$. From the proof of Claim 3.7, we get $\gamma_{p a}\left(T^{\prime}\right) \leq \frac{3 n^{\prime}-2 l^{\prime}-s^{\prime}+2}{5}$. Thus, the equality holds by Theorem 2.4. Using the inductive hypothesis, we deduce that $T^{\prime} \in \mathfrak{F}$. Thus $T \in \mathfrak{F}$ since it is obtained from $T^{\prime}$ by applying operation $O$.

Case 2. $d_{T}\left(u_{2}\right)=2$.

Claim 3.8. $d_{T}\left(u_{3}\right) \geq 3$.

Proof. Suppose $d_{T}\left(u_{3}\right)=2$. Either $u_{3}$ or $u_{4}$ is in $D$. Without loss of generality, we suppose that $u_{4} \in D$ as we may replace $u_{3}$ in $D$ by $u_{4}$. Let us consider $T^{\prime}=T-T_{u_{2}}$ (see (a) in Fig. 7). It is easy to check that if $T^{\prime}=S_{p}, 1 \leq p \leq 3$ or $T^{\prime}=P_{4}$ then $\gamma_{p a}(T) \in\{3,4\}>\frac{3 n-2 l-s+2}{5}$. So, $n^{\prime} \geq 5$. Clearly, $D \cap V\left(T^{\prime}\right)$ is a gpa of $T^{\prime}$ implying that $\gamma_{p a}\left(T^{\prime}\right) \leq \gamma_{p a}(T)-2=\frac{3 n-2 l-s+2}{5}-2$. We have $n=n^{\prime}+2+\left|L_{u_{1}}\right|$. If $u_{4}$ is a support vertex then $s=s^{\prime}+1$ and $l=l^{\prime}-1+\left|L_{u_{1}}\right|$. Thus, $\gamma_{p a}\left(T^{\prime}\right) \leq \frac{3 n^{\prime}-2 l^{\prime}-s^{\prime}+2}{5}+\frac{\left|L_{u_{1}}\right|-3}{5}$. By Claim 3.4, $\left|L_{u_{1}}\right| \leq 2$ then $\gamma_{p a}\left(T^{\prime}\right) \leq \frac{3 n^{\prime}-2 l^{\prime}-s^{\prime}+2}{5}-\frac{1}{5}<\frac{3 n^{\prime}-2 l^{\prime}-s^{\prime}+2}{5}$ which contradicts Theorem 2.4. Suppose now that $u_{4}$ is not a support vertex, so $s=s^{\prime}$ and $l=l^{\prime}-1+\left|L_{u_{1}}\right|$. It follows that $\gamma_{p a}\left(T^{\prime}\right) \leq \frac{3 n^{\prime}-2 l^{\prime}-s^{\prime}+2}{5}+\frac{\left|L_{u_{1}}\right|-2}{5}$. Since $\left|L_{u_{1}}\right| \leq 2$, if $\left|L_{u_{1}}\right|=1$, we get $\gamma_{p a}\left(T^{\prime}\right)<\frac{3 n^{\prime}-2 l^{\prime}-s^{\prime}+2}{5}$ which contradicts Theorem 2.4. So $\left|L_{u_{1}}\right|=2$ and then $\gamma_{p a}\left(T^{\prime}\right) \leq \frac{3 n^{\prime}-2 l^{\prime}-s^{\prime}+2}{5}$. We get the equality by Theorem 2.4 and using the induction hypothesis, we deduce that $T^{\prime} \in \mathfrak{F}$. So, by Observation 3.1, every non-leaf vertex of $T^{\prime}$ is a strong support vertex. Thus, $u_{4}$ is a support vertex in $T$ which contradicts our assumption.

Claim 3.9. $\left|N\left[u_{3}\right] \cap D\right|=\left|N\left[u_{3}\right]-D\right|$.

Proof. By Claim 3.8, $d_{T}\left(u_{3}\right) \geq 3$. Each child of $u_{3}$ is either a leaf or a support vertex or a non-leaf neighbor of a support vertex. Assume that $\left|N\left[u_{3}\right] \cap D\right|>\left|N\left[u_{3}\right]-D\right|$. Let us consider $T^{\prime}=T-T_{u_{2}}$ (see (b) in Fig. 7). We can easily check that $T^{\prime} \neq S_{p}, 2 \leq p \leq 3$ and $T^{\prime} \neq P_{4}$ otherwise $\gamma_{p a}(T)>\frac{3 n-2 l-s+2}{5}$ which contradicts the assumption on $T$. So $n^{\prime} \geq 5$. Clearly, $D \cap V\left(T^{\prime}\right)$ is a gpa of $T^{\prime}$ implying that $\gamma_{p a}\left(T^{\prime}\right) \leq \gamma_{p a}(T)-2=\frac{3 n-2 l-s+2}{5}-2$. Since $n=n^{\prime}+2+\left|L_{u_{1}}\right|, l=l^{\prime}+\left|L_{u_{1}}\right|$ and $s=s^{\prime}+1$, we obtain $\gamma_{p a}\left(T^{\prime}\right) \leq \frac{3 n^{\prime}-2 l^{\prime}-s^{\prime}+2}{5}+\frac{\left|L_{u_{1}}\right|-5}{5}$. By Claim 3.4, $\left|L_{u_{1}}\right| \leq 2$ then $\gamma_{p a}\left(T^{\prime}\right) \leq \frac{3 n^{\prime}-2 l^{\prime}-s^{\prime}+2}{5}-\frac{3}{5}<\frac{3 n^{\prime}-2 l^{\prime}-s^{\prime}+2}{5}$ which contradicts Theorem 2.4.

Claim 3.10. $u_{3}$ is a support vertex.

Proof. Assume that $u_{3}$ is not a support vertex. So, by the minimality of $D$ and Observation 2.3, every child of $u_{3}$ is in $D$ as it is either a support vertex or a non-leaf neighbor of a support vertex. Consequently, if $u_{3} \in D$ then $\left|N\left[u_{3}\right] \cap D\right|>\left|N\left[u_{3}\right]-D\right|$ (see (b) in Fig. 7) which contradicts Claim 3.9. So, $u_{3} \notin D$ and according to Claim 3.9, $d_{T}\left(u_{3}\right)=3$ and $u_{4} \notin D$ (see (a) in Fig. 8). Then $u_{4}$ is different from a support vertex, otherwise, by the minimality of $D$ and Observation 2.3, $u_{4}$ will be in $D$. Let $v, u$ be the children of $u_{3}$ and $v$, respectively in $T_{u_{3}}$. If $v$ is a support vertex then $\left|L_{v}\right|=1$ otherwise $u_{3}$ will be in $D$ as it may replace any leaf of $v$ in $D$. But the 


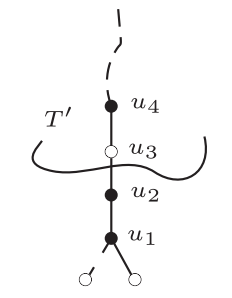

(a) $: d_{T}\left(u_{3}\right)=2$. (b) : $d_{T}\left(u_{3}\right) \geq 3,\left|N\left[u_{3}\right] \cap D\right|>\left|N\left[u_{3}\right]-D\right|$.

Figure 7 . In (b), $u_{3}$ may be a support. In both cases (a) and (b), $T^{\prime}=T-T_{u_{2}}$.

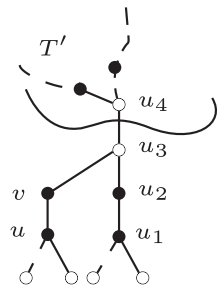

(a)

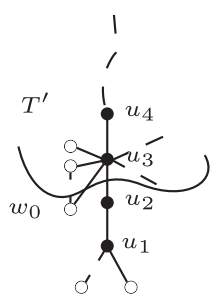

(b)

FiguRE 8. In (a): $u_{3}, u_{4} \notin D, T^{\prime}=T-T_{u_{3}}$. In (b), $u_{3}$ is a strong support vertex and $T^{\prime}=$ $T-T_{u_{2}}-\left\{w_{0}\right\}$.

weakness of $v$ contradicts our remark given above about the non-pendant support vertices which must be strong. So, $v$ is not a support vertex. Let us consider $T^{\prime}=T-T_{u_{3}}$. Since $u_{4} \notin D$, then $u_{4}$ has at least two neighbors in $D \cap V\left(T^{\prime}\right)$. The vertex $u_{t}$ cannot be the parent of $u_{4}$, otherwise $u_{4} \in D$ as it may replace $u_{t}$ in $D$. Further, $u_{4}$ cannot be adjacent to a leaf or a support vertex, otherwise $u_{4}$ is in $D$ as it is a support or it can replace any leaf in $D$ of its support neighbor. It follows that $\operatorname{diam}\left(T^{\prime}\right) \geq 4$ and $n^{\prime} \geq 5$. The set $D \cap V\left(T^{\prime}\right)$ is a gpa of $T^{\prime}$ implying that $\gamma_{p a}\left(T^{\prime}\right) \leq \gamma_{p a}(T)-4=\frac{3 n-2 l-s+2}{5}-4$. Since $n=n^{\prime}+5+\left|L_{u_{1}}\right|+\left|L_{u}\right|, l=l^{\prime}+\left|L_{u_{1}}\right|+\left|L_{u}\right|$ and $s=s^{\prime}+2$, we obtain $\gamma_{p a}\left(T^{\prime}\right) \leq \frac{3 n^{\prime}-2 l^{\prime}-s^{\prime}+2}{5}+\frac{\left|L_{u_{1}}\right|+\left|L_{u}\right|-7}{5}$. By Claim 3.4, $\left|L_{u_{1}}\right|+\left|L_{u}\right| \leq 4$, we get $\gamma_{p a}\left(T^{\prime}\right)<\frac{3 n^{\prime}-2 l^{\prime}-s^{\prime}+2}{5}$ which contradicts Theorem 2.4 .

It follows from the previous Claims and the remark given above that $u_{3}$ is a strong support vertex verifying $\left|N\left[u_{3}\right] \cap D\right|=\left|N\left[u_{3}\right]-D\right|$. Let $w_{0}$ be a leaf of $u_{3}$ not in $D$. Let us consider $T^{\prime}=T-\left(T_{u_{2}} \cup\left\{w_{0}\right\}\right)$ (see (b) in Fig. 8). It is easy to check that $u_{t}$ cannot be the parent of $u_{3}$ otherwise $T^{\prime}=S_{3}$ and then $\gamma_{p a}(T)=4$. So $\gamma_{p a}(T)>\frac{3 n-2 l-s+2}{5}$ which is a contradiction. So, $n^{\prime} \geq 5$. Clearly, $D \cap V\left(T^{\prime}\right)$ is a gpa of $T^{\prime}$ implying that $\gamma_{p a}\left(T^{\prime}\right) \leq \gamma_{p a}(T)-2=\frac{3 n-2 l-s+2}{5}-2$. Since $n=n^{\prime}+3+\left|L_{u_{1}}\right|, l=l^{\prime}+1+\left|L_{u_{1}}\right|$ and $s=s^{\prime}+1$ we get $\gamma_{p a}\left(T^{\prime}\right) \leq \frac{3 n^{\prime}-2 l^{\prime}-s^{\prime}+2}{5}+\frac{\left|L_{u_{1}}\right|-4}{5}$. By Claim 3.4, $\left|L_{u_{1}}\right| \leq 2$ and then $\gamma_{p a}\left(T^{\prime}\right) \leq \frac{3 n^{\prime}-2 l^{\prime}-s^{\prime}+2}{5}-\frac{2}{5}<\frac{3 n^{\prime}-2 l^{\prime}-s^{\prime}+2}{5}$ which contradicts Theorem 2.4 and the proof is complete.

\section{Conclusion}

We give in this paper a lower bound on the global powerful alliance number of any tree in terms of its order and its numbers of leaves and support vertices. Moreover, we characterize all extremal trees attaining this bound. Bouzefrane [1] shows that any tree $T$ different from a star $S_{p}$ with order $n \geq 4, l$ leaves and $s$ support vertices verifies $\gamma_{p a}(T) \leq \frac{4 n-l+s}{6}$. The first author of this paper characterizes all extremal trees achieving this 
bound in [9]. Thus, we obtain a framing of the global powerful alliance number in the class of trees. Among the open problems raised by our results, the following are of particular interest.

- Explore the bounds on the global powerful alliance number in particular classes of graphs like the unicycle graphs, bipartite ones and the cactus.

- Characterize trees with a unique minimum global powerful alliance.

Acknowledgements. The authors are grateful to anonymous referees for their remarks and suggestions that helped improve the manuscript.

\section{REFERENCES}

[1] M. Bouzefrane, On alliances in graphs, Magister memory. University of Blida, Algeria (2010).

[2] R.C. Brigham, R.D. Dutton, T.W. Haynes and S.T. Hedetniemi, Powerful alliances in graphs. Discrete Math. 309 (2009) $2140-2147$.

[3] A. Cami, H. Balakrishnan, N. Deo and R.D. Dutton, On the complexity of finding optimal global alliances. J. Combin. Math. Combin. Comput. 58 (2006) 23-31.

[4] M. Chellali, Offensive alliances in bipartite graphs. J. Combin. Math. Combin. Comput. 73 (2010) 245-255.

[5] M. Chellali and T. Haynes, Global alliances and independence in trees. Discuss. Math. Graph Theory 27 (2007) $19-27$.

[6] O. Favaron, G. Fricke, W. Goddard, S.M. Hedetniemi, S.T. Hedetniemi, P. Kristiansen, R.C. Laskar and D.R. Skaggs, Offensive alliances in graphs. Discuss. Math. Graph Theory 24 (2004) 263-275.

[7] A. Harutyunyan, A fast algorithm for powerful alliances in trees. In: International Conference on Combinatorial Optimisation and Applications, COCOA. (2010) 31-40.

[8] S.M. Hedetniemi, S.T. Hedetniemi and P. Kristiansen, Alliances in graphs. J. Combin. Math. Combin. Comput. 48 (2004) $157-177$.

[9] S. Ouatiki, On the upper global powerful alliance number in trees. Accepted in Ars Combinatoria. 